\title{
Structural analysis and insight into novel MMP-13 inhibitors from natural chemiome as disease-modifying osteoarthritis drugs
}

\author{
Yang Xiang ${ }^{1}$, Yuanhui Li ${ }^{1}$, Zhirong Ling ${ }^{1}$, Yanzi Cheng ${ }^{2 \star}$ \\ ${ }^{1}$ Department of Orthopaedics, ${ }^{2}$ Department of Intensive Care Unit, Zhuhai People's Hospital, Jinan University Affiliated Zhuhai \\ Hospital, Zhuhai, Guangdong, China \\ *For correspondence: Email: CTyronehott@yahoo.com
}

Sent for review: 3 May 2018

Revised accepted: 22 October 2018

\begin{abstract}
Purpose: To identify natural chemiome that inhibits matrix-metalloproteinases (MMPs) with a view to discovering novel disease-modifying osteoarthritis drugs (DMOADs).

Methods: Computer-aided drug design (CADD) with virtual screening, ADME/Tox, molecular docking, molecular dynamics simulation, and MM-PBSA calculations were used in search of novel natural compounds that inhibit MMPs.

Results: From more than fifty thousand compounds, a single lead compound (IBS ID: 77312) was shortlisted using bias based on binding energy and drug-likeness. This lead compound synergistically bound to the S1 domain of MMP-13 protein through five hydrogen bonds. The interactions became stable within 100-nanosecond molecular dynamics simulation run. The in vitro data for the lead compound showed that its minimal non-lethal dose increased collagen content but decreased aggrecan level in chondrocytes.

Conclusion: This study has identified a natural lead compound that may pave the way for a novel $D M O A D$ of natural origin against $O A$.
\end{abstract}

Keywords: Osteoarthritis, MMP-13, Natural chemiome, Disease-modifying osteoarthritis drug, Molecular docking

\footnotetext{
This is an Open Access article that uses a funding model which does not charge readers or their institutions for access and distributed under the terms of the Creative Commons Attribution License (http://creativecommons.org/licenses/by/4.0) and the Budapest Open Access Initiative (http://www.budapestopenaccessinitiative.org/read), which permit unrestricted use, distribution, and reproduction in any medium, provided the original work is properly credited.

Tropical Journal of Pharmaceutical Research is indexed by Science Citation Index (SciSearch), Scopus, International Pharmaceutical Abstract, Chemical Abstracts, Embase, Index Copernicus, EBSCO, African Index Medicus, JournalSeek, Journal Citation Reports/Science Edition, Directory of Open Access Journals (DOAJ), African Journal Online, Bioline International, Open-J-Gate and Pharmacy Abstracts
}

\section{INTRODUCTION}

Osteoarthritis (OA) is an autoimmune degenerative disorder and the main cause of physical disability and poor quality of life in industrialized countries [1]. The disease which affects the joints, is characterized by irreversible damage to articular cartilage, and several studies have so far focused on the molecular biology of the events that lead to cartilage erosion [2]. The loss of articular cartilage in $\mathrm{OA}$ is triggered by gradual degradation and fibrillation of the cartilage surface. The etiology of articular cartilage degradation lies in the damage to type II collagen [3]. Type II collagen, a structural protein which is affected in OA patients, is considered being as one of the main prime targets for new anti-OA drug discovery. There is usually a strict balance between collagen type II production and its degradation by catabolic enzymes. This 
balance is disrupted due to increased protein degradation [4]. Matrix metalloproteinases (MMPs) are catabolic enzymes responsible for collagen type II degradation [5].

Disease-modifying OA drugs (DMOADs) are compounds capable of delaying or halting the progressive destruction of joint tissuein OA. These drugs can be developed through advancements in the understanding of the pathophysiology of $\mathrm{OA}$, and identification of promising therapeutic targets [6]. Human-3 collagen (MMP-13) has been widely reported as a target for development of DMOADs [7]. In mice deficient in MMP-13, OA cartilage erosion is reduced [8]. Human-3 collagen (MMP-13) belongs to the family of zinc-dependent enzymes that are assembled in synovial cells and chondrocytes. It is responsible for decomposing cellular matrix collagen type II protein and maintaining cartilage-specific matrix phenotype [9-11].

So far, a few compounds have been reported as DMOADs, but no studies have investigated natural compounds as DMOADs against MMP13 [12, ]. In order to identify DMOADs of natural origin, a natural product database (interBioScreen) that includes about sixty-six thousand compounds was selected. In this database, $60-65 \%$ of the compounds are of plant origin, $5-10 \%$ are of microbial origin, $5 \%$ are from marine species, while the rest are from other natural sources. In this study, in silico tools were used to search for natural DMOADs against MMP-13. Virtual Screening (VS) of sixty-six thousand compounds gave $<1 \%$ of the compounds for further evaluation on druglikeness parameters. Eight compounds were subjected to molecular docking simulations against the active site of MMP-13. The top lead compounds were evaluated at atomic level using 100 nanosecond (ns) molecular dynamics simulation with GROMACS atomic simulation tool. The trajectory from the simulations was used for molecular mechanics-PoissonBoltzmann surface area (MM-PBSA) analysis, for understanding the binding mode of the top lead compound against MMP-13. The top lead compound was exposed to rat chondrocytes under in vitro conditions.

\section{EXPERIMENTAL}

\section{Protein and compound database preparation}

MMP-13 protein in complex with 2napthylsulfonamide hydroxamic acid inhibitor downloaded from RCSB protein databank (PDB ID $3 Z X H, 1.30 \AA$ x-ray resolution) was used in this study. The structure is a dimer and its monomer from104-272 amino acids was used. The structure was stripped of 2-napthylsulfonamide hydroxamic acid inhibitor and water molecules, and the missing atoms in some of the amino acids in the selected crystallographic structure were added. Energy minimization was done with SPDB viewer. Using OPLS 2001 force field, a final root mean square deviation (RMSD) value of $0.3 \AA$ was obtained. The natural compound database used in this study was downloaded from interBioScreen website (https://www. ibscreen.com/).

\section{Virtual screening for prediction of drug likeliness}

Virtual screening was done using ArgusLab suit which limited the number of compounds based on binding energy bias. The S1 binding region of the catalytic domain was selected for screening of natural compounds. For shortlisting, only compounds showing binding energies less than $8 \mathrm{kcal} / \mathrm{mol}$ were selected for further studies. Less than $1 \%$ of the total compounds were shortlisted, based on their binding energy $(\Delta G)$ calculations. The selected compounds were further limited by Lipinki Rule of five (RO5) [13]. The RO5 parameters gave eight compounds for molecular docking.

\section{Molecular docking simulations}

The shortlisted eight compounds were subjected to atomic scale target-based drug discovery through molecular docking simulation performed using AutoDocksuit (V. 4.2.6) [14]. AutoDock suit calculates binding free energy for the best protein-compound complex. The suit uses the binding location definition in the form of a grid to create a rectangular network, and the dockings of the compound are usually performed within this rectangular network. AutoDock suit calculates energy values which represent the total energy of the protein, the total energy of the compound and the total torsional free energy. In this study, the results were generated by setting the AutoDock suit to the default parameters, where the maximum ratings were evaluated to illustrate the top ten binding styles per run. Docking results were analyzed using Discovery Studio Visualizer. The best combination of natural compound with MMP-13 was selected for further assessment using molecular dynamic simulation tool.

\section{Molecular dynamics simulation}

The top lead compound for DMOAD against MMP-13 was studied for stability and dynamic 
interaction using GROMACS atomic simulation platform over 100 nanosecond (ns) time period [15]. The protein was subjected to GROMOS 43a1 force field, and force field of the compound was calculated by using online PRODRG web server $[16,17]$. A water bath model with simple point charge (SPC216) was used for water molecules in this simulation. The whole system was energy-minimized by NVT and NPT ensembles, which are governed by Berendsen coupling scheme. For NVT ensemble, a constant temperature of $300 \mathrm{~K}$ with $1 \mathrm{~ns}$ time interval and 0.1 ps coupling constant was used. This step was followed by a NPT ensemble where 1 ns time interval and constant pressure of 1 bar with constant coupling of 5 ps was used. A 100-ns simulation of the complex at $300 \mathrm{~K}$ was carried out using SHAKE algorithm and particle mesh Ewald method. In Ewald method, a 14-Å cutoff for van der Waals interactions, and a 12- $\AA$ cutoff for Coulomb interaction were updated after every 2 steps [18]. Pymol, Ligplus and VMD tools were used for data analysis. The graphs were plotted using Grace program.

\section{MM-PBSA calculations}

The MMP-13 and lead complex interaction at residue level were used for MM-PBSA calculations, based on the trajectory from the simulation [19]. For MMP-13 and lead complex trajectory, 1000 snapshots were taken, and each snapshot was retrieved after 100ps. The binding mode of MMP-13 with the natural lead compound was studied using grace plotting software. The binding energy $\left(\Delta G_{\text {bind }}\right)$ of MMP-13 protein with the shortlisted natural compound in water was calculated with MM-PBSA using Eq 1.

$\Delta G_{\text {bind }}=G_{c}-\left(G_{P}+G_{L}\right)$

Where $G_{c}$ is total free energy of MMP-13 and lead complex, while $G_{P}$ and $G_{L}$ are the total free energies of MMP-13 protein and compound in water, respectively. The free energy for MMP-13 and natural compound was obtained as in Eq 2.

$G_{x}=E_{m m}-T S+G_{s o l v}$

where $G_{x}$ represents free energy of MMP-13 or natural compound, $E_{m m}$ is their mean molecular mechanics potential energy in vacuum, TS represents entropic contribution to $E_{m m}$ in vacuum, and $G_{\text {solv }}$ is the free energy of solvation.

\section{Isolation and culture of chondrocytes}

Female Sprague-Dawley rats weighing about $120 \mathrm{~g}$ were purchased from the Laboratory Animal Center of Xi'an Jiaotong University (Xi'an,
China). Fetal bovine serum (FBS) and DMEM were purchased from Invitrogen. Hyaluronidase, collagenase, trypsin, and alcian blue 8GX were products of Sigma. Mouse anti-rat collagen monoclonal antibody from Neomarker. Goat antirat aggrecan monoclonal antibody was obtained from Santa Cruz Biotechnology. DAB kit was purchased from Beijing Zhongshan, RNA isolation kit was product of Shanghai Feijie, while first strand CDNA synthetic kit was obtained from MBI.

The isolation and culture of rat costal chondrocytes were performed as described previously [20]. The rats were sacrificed by cervical dislocation. Their ribs were removed and the cartilage was collected and cut into $1-\mathrm{mm}^{3}$ portions. The tissue samples were digested with hyaluronidase and collagenase, and the isolated cells were cultured at $37^{\circ} \mathrm{C}$ under a humidified atmosphere containing $5 \% \mathrm{CO}_{2}$. The cells were sub-cultured at a ratio of $1: 1$ after treatment with $0.25 \%$ trypsin/EDTA. The isolated chondrocytes were identified by immunostaining for collagen and aggrecan. In this study, the cells were used at passage two.

\section{Treatment of chondrocytes with top lead compound}

Cells $\left(5 \times 10^{4}\right)$ were seeded in triplicates in 24well plates and incubated with different concentrations of lead compound (1,10 and 100 $\mu \mathrm{M})$ for $48 \mathrm{~h}$. The cells were then collected and tested for expression.

\section{Immunocytochemical analysis}

Cells were placed on coverslips and were grown to $80 \%$ confluence. They were washed and fixed in $4 \%$ paraformaldehyde for $30 \mathrm{~min}$, and nonspecific binding sites were blocked with normal goat serum. The cells were then incubated at $4^{\circ} \mathrm{C}$ overnight with anti-aggrecan (1:200 dilution) and anti-collagen (1:200 dilution) antibody, followed by incubation with biotinylated IgG (1:400 dilution). The bound antibody was visualized using 3,3'-diaminobenzidine. Coverslips were mounted and the cells were examined under a microscope.

\section{RESULTS}

\section{Drug likeliness}

The methods used in this study are shown in Figure 1. The S1 pocket of the MMP-13 protein is the active cleft (site) used for virtual screening of the sixty-six thousand compounds of natural origin. The $\mathrm{S} 1$ pocket has the active site cleft 
which is the continuation of the catalytic domain in $\beta$-sheet conformation, forming hydrogen bonds with S1 pocket wall Pro242.

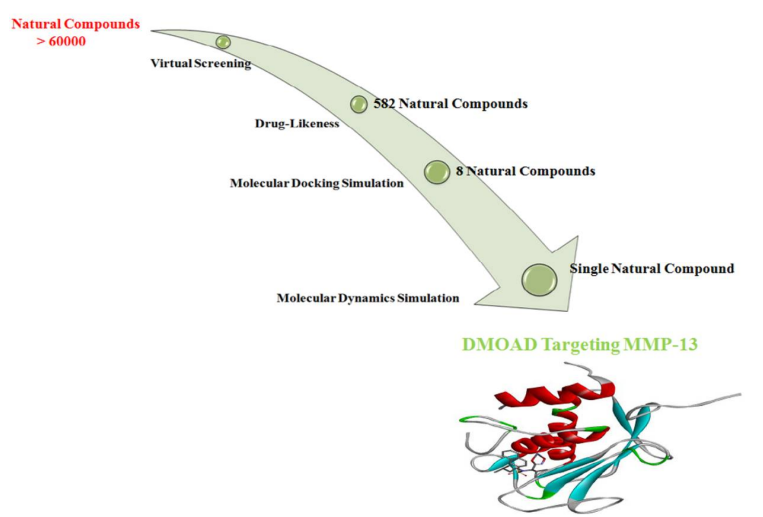

Figure 1: Methodology used to find a novel lead of natural origin against osteoarthritis

Figure 2 a shows the pocket selected for virtual screening with a possible DMOAD in the cavity. The virtual screening grid (Figure 2 b) was created around the $S 1$ pocket and flexible docking was used for shortlisting the compounds. Binding energy score was used to shortlist compounds for further DMOAD development. For this, a binding energy bias was set where only compounds with $\Delta \mathrm{G}$ less than $-9 \mathrm{kcal} / \mathrm{mol}$ were shortlisted. The maximum binding energy shown in this screening was $-13.765 \mathrm{kcal} / \mathrm{mol}$, and only 582 natural compounds showed binding energies in the range of -9 to $-13.765 \mathrm{kcal} / \mathrm{mol}$. The selected compounds were further shortlisted by checking them with parameters set by Lipinski, where four properties i.e. molecular weight (MW), octanol/water partition coefficient (clogP), hydrogen bond donor (HBD) and hydrogen bond acceptor (HBA) were evaluated. The method is an established procedure in lead development. Indeed, 90\%of the oral drugs that passed phase Il clinical trial follow RO5 parameters [17].

The shortlisted compounds were further checked for toxicity, and only compounds which were noncarcinogenic and non-mutagenic were shortlisted. After drug likeliness prediction analysis, eight compounds were subjected to molecular docking simulation analysis (Figure 3).

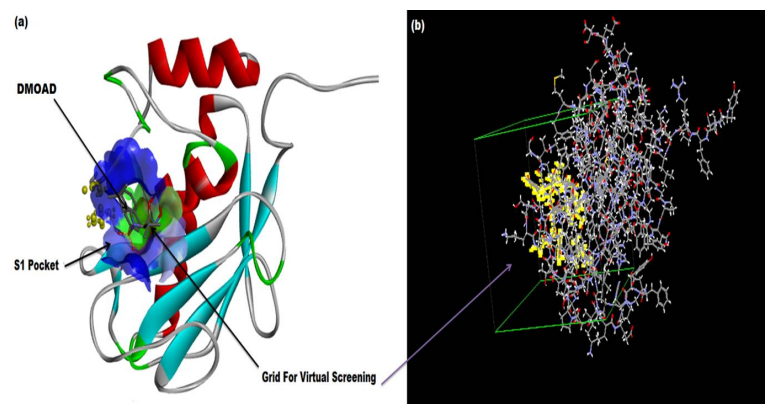

Figure 2: (a) The S1 pocket of MMP-13 protein used to design DMOAD of natural origin; (b) the grid around Si domain used for virtual screening

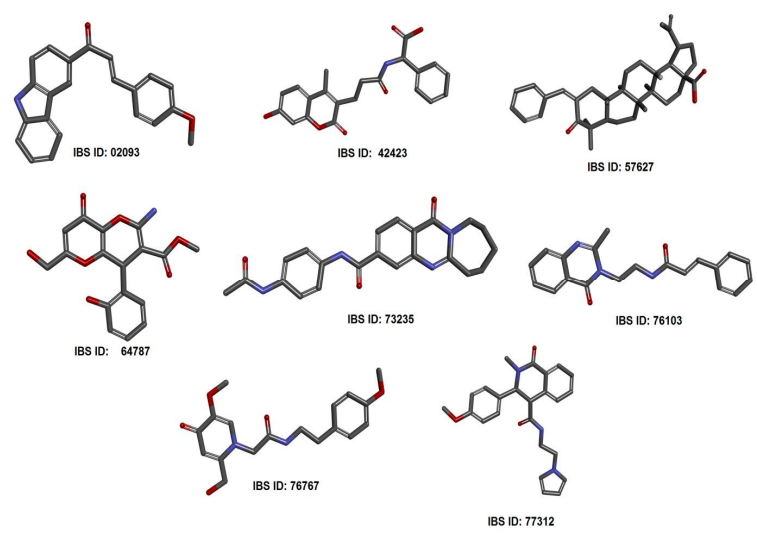

Figure 3: Top eight natural compounds inhibiting MMP-13

\section{Molecular docking simulation}

Details of the eight compounds selected for atomic scale DMOAD development against MMP-13 protein are given in Table 1.

Each compound was subjected to docking by AutoDock docking tool into the optimized S1 active pocket of MMP-13 protein. Maximum evaluations were performed for each docking and top ten binding poses were generated for each compound.

Table 1: Drug-likeliness of top Eight lead compounds

\begin{tabular}{lllllll}
\hline IBS No & clogp & HBD & HBA & tpsa & Mutagenicity & Carcinogenicity \\
\hline 02093 & 5.223 & 1 & 3 & 42.09 & $\mathrm{NO}$ & $\mathrm{NO}$ \\
42423 & 2.758 & 3 & 7 & 54.37 & $\mathrm{NO}$ & $\mathrm{NO}$ \\
57627 & 10.418 & 1 & 3 & 116.84 & $\mathrm{NO}$ & $\mathrm{NO}$ \\
64787 & 0.472 & 3 & 8 & 132.22 & $\mathrm{NO}$ & $\mathrm{NO}$ \\
73235 & 2.297 & 2 & 7 & 93.09 & $\mathrm{NO}$ & $\mathrm{NO}$ \\
76103 & 3.179 & 1 & 5 & 63.99 & $\mathrm{NO}$ & $\mathrm{NO}$ \\
76767 & -0.75 & 2 & 7 & 89.79 & & \\
77312 & 3.189 & 1 & 6 & 63.57 & & \\
\hline
\end{tabular}


The top generated pose of each compound was studied using Discovery Studio Visualizer and the results are shown in Table 2. Two compounds out of eight formed hydrogen bonds with S1 pocket of MMP-13 protein.

\section{MMP-13 S1 pocket jammers}

Two natural compounds (Table 2) were found to jam the S1 pocket of MMP-13 protein with noncovalent hydrogen bond interactions.

The first shortlisted compound was IBS ID: 64787 , and its interaction with $S 1$ pocket is displayed in Figure 4.This compound showed $\Delta G$ of $-8.23 \mathrm{kcal} / \mathrm{mol}$, and it jammed the $S 1$ pocket through formation of two hydrogen bonds with Pro242and Tyr244. The binding pocket for the compound was formed by the amino acids His187, His226, Glu223, Ala186, Leu184, Leu185, His232, His222, Tyr244, Val219, Phe 241, Ile243 and Pro242. The oxygen at first position (O-1) of the compound formed hydrogen bond with Pro242 and the hydrogen bond length was $2.53 \mathrm{~A}^{\circ}$.

The oxygen at third position (O-3) of the compound formed one hydrogen bond of $1.92 \mathrm{~A}^{\circ}$ length with Tyr244. The mapping of IBS ID: 64787 against the hit (Pro242and Tyr244) is shown in Figure 5a. The second best compound of natural origin was IBS ID:77312 which interacted with the active site of MMP-13 through 5 hydrogen bonds. Its $\Delta$ Gwas $-9.67 \mathrm{kcal} / \mathrm{mol}$, and its binding pocket comprisedHis187, His226, Glu223, Ala186, Leu184, Leu185, His232, His222, Tyr244, Val219, Phe 241, lle243 and
Pro242. The details are shown in Table 3. Figure 5 shows the mapping of the five hits by IBS ID:77312. Based on the molecular docking simulation analysis, IBS ID:77312 was selected as the lead compound for the development of DMOAD.

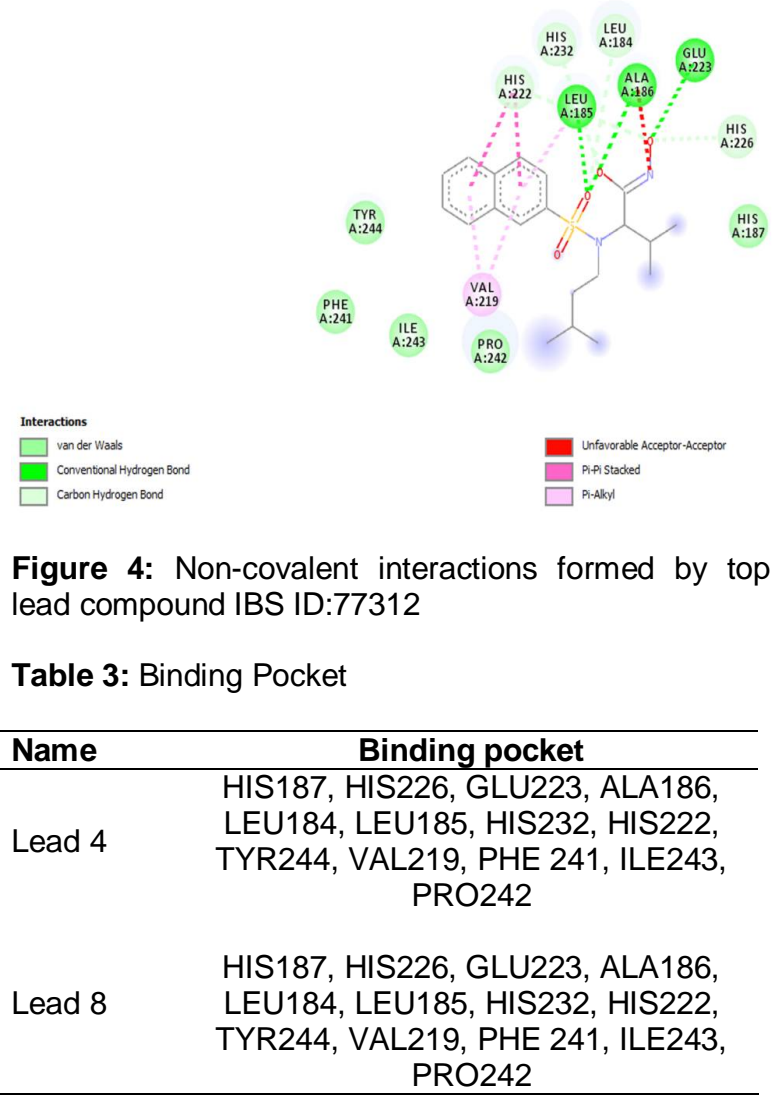

Table 2: Binding pose analysis of top eight compounds, the ligand binding pocket and the hydrogen bond formation was calculated using Discovery Studio v 16.1.015350

\begin{tabular}{|c|c|c|c|c|}
\hline Name & IBS ID & $\begin{array}{c}\Delta \mathbf{G} \\
\mathrm{Kcal} / \mathrm{mol}\end{array}$ & IUPAC name & H-bonds \\
\hline Lead 1 & 02093 & -5.23 & (E)-1-(9H-carbazol-3-yl)-3-(4-methoxyphenyl)prop-2-en-1-one & \\
\hline Lead 2 & 42423 & -8.12 & $\begin{array}{l}\text { 2-(3-(7-hydroxy-4-methyl-2-oxo-2H-chromen-3-yl)propanamido)-2- } \\
\text { phenylacetic acid }\end{array}$ & \\
\hline Lead 3 & 57627 & -3.12 & $\begin{array}{l}\text { (1R,3aS,5aR,5bR,11aR,11bR,13aR,Z)-10-benzylidene- } \\
\text { 5a,5b,8,8,11a-pentamethyl-9-oxo-1-(prop-1-en-2-yl)icosahydro-1H- } \\
\text { cyclopenta[a]chrysene-3a-carboxylic acid }\end{array}$ & \\
\hline Lead 4 & 64787 & -8.23 & $\begin{array}{l}\text { methyl 2-amino-6-(hydroxymethyl)-4-(2-hydroxyphenyl)-8-oxo-4,8- } \\
\text { dihydropyrano[3,2-b]pyran-3-carboxylate }\end{array}$ & Two \\
\hline Lead 5 & 73235 & -4.23 & $\begin{array}{l}\mathrm{N} \text {-(4-acetamidophenyl)-12-oxo-6,7,8,9,10,12-hexahydroazepino[2,1- } \\
\text { b]quinazoline-3-carboxamide }\end{array}$ & \\
\hline Lead 6 & 76103 & -7.13 & $\mathrm{~N}$-(2-(2-methyl-4-oxoquinazolin-3(4H)-yl)ethyl)cinnamamide & \\
\hline Lead 7 & 76767 & -8.17 & $\begin{array}{l}\text { 2-(2-(hydroxymethyl)-5-methoxy-4-oxopyridin-1(4H)-yl)-N-(4- } \\
\text { methoxyphenethyl)acetamide }\end{array}$ & \\
\hline Lead 8 & 77312 & -9.67 & $\begin{array}{l}\mathrm{N}-(2-(1 \mathrm{H} \text {-pyrrol-1-yl)ethyl)-3-(4-methoxyphenyl)-2-methyl-1-oxo- } \\
\text { 1,2,3,4-tetrahydroisoquinoline-4-carboxamide }\end{array}$ & Five \\
\hline
\end{tabular}




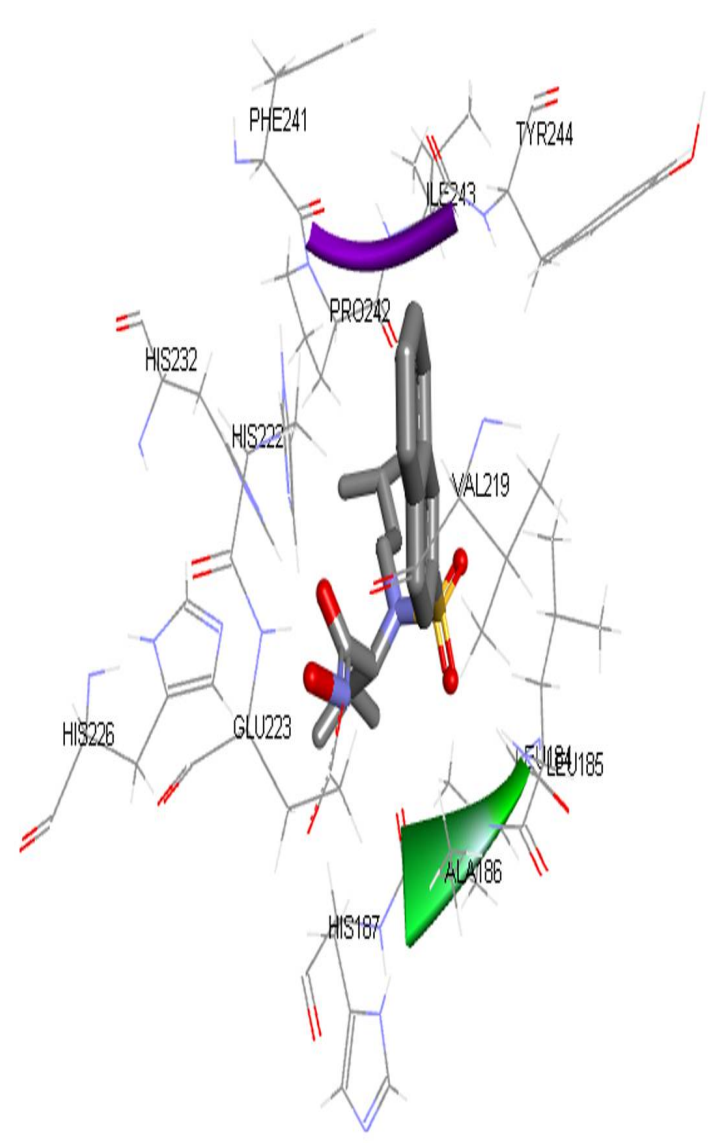

Figure 5: Binding pocket of top lead compound with si pocket of MMP-13 protein.

\section{Molecular dynamics simulation}

Molecular dynamics simulation was carried out for the dynamic analysis of the complex to monitorlead-MMP13 complex interaction over time, and 100 ns MDS was done using GROMOS 43a1 force field. The lead-MMP13 complex was stable throughout the run. Root mean square deviation (RMSD) was used to evaluate the MMP-13 protein structure convergence to equilibrium after the interaction of the compound. The backbone atoms of MMP13 were used to evaluate the RMSD plot (Figure 6a). The complex converged with RMSD range almost above $0.4 \mathrm{~nm}$. The hydrogen bond plot results showed a range of 1 to 7 hydrogen bonds formed in 100 ns simulation (Figure 6b). The hydrogen bond formation showed strong binding of the shortlisted compound with MMP-13.

\section{MM/PBSA}

The MDS trajectory of the lead compound and MMP-13 protein was used for MM-PBSA to evaluate binding free energy over a period of 100ns. The complex of protein and the compound was taken after every 100-ps of stable intervals from the trajectory and were used to calculate the binding free energy. The MM-PBSA results indicated that the lead compound possessed a negative binding free energy of $244.764 \mathrm{~kJ} / \mathrm{mol}$. The relative binding free energies of the complex of lead-MMP13 showed strong binding in the dynamic system. The interaction with $\mathrm{S} 1$ pocket of MMP13 is shown in Figure 7.

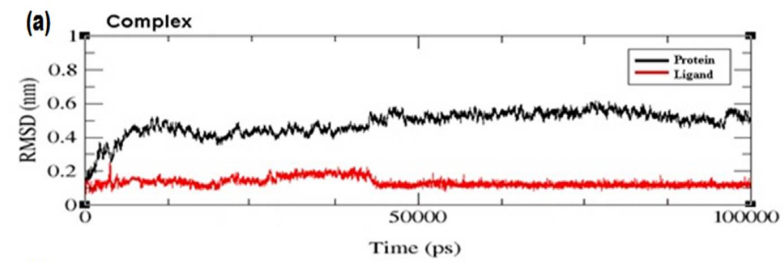

(b)

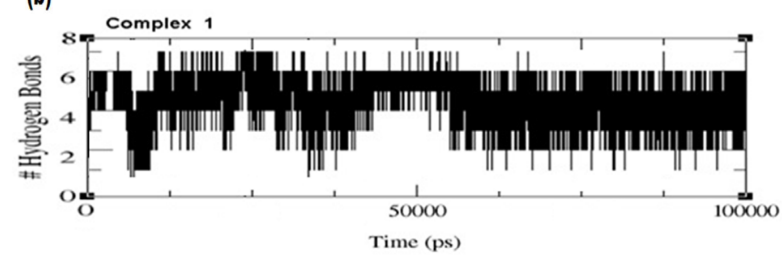

Figure 6: (a) RMSD plot of the top complex over 100ns simulation, black represents the protein and red represents lead compound IBS ID:77312; (b) The hydrogen bond plot between ligand and protein showing the number varying from one to seven hydrogen bonds over a period of $100 \mathrm{~ns}$

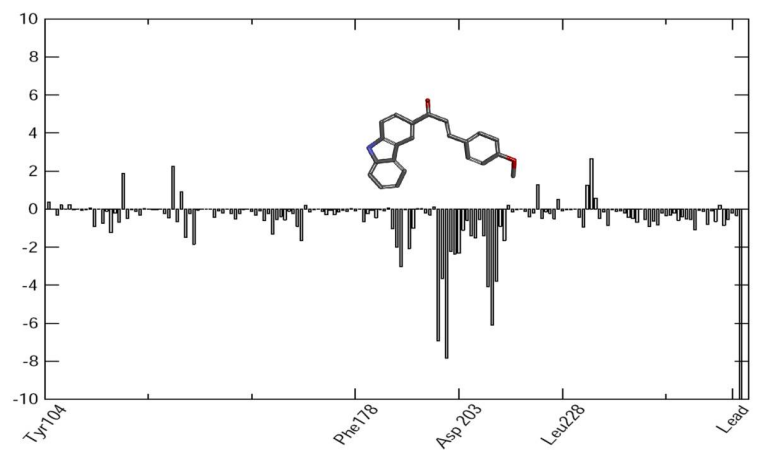

Figure 7: Binding energy distribution per residue showing the binding of ligand with S1 pocket of MMP13 protein

\section{Morphological and immunocytochemical findings}

The isolated cells attached to the culture plate within $24 \mathrm{~h}$ and showed a typical polygon shape. After culturing for 3 - 5 days, cells grew to confluence and could be sub-cultured. The amount of aggrecan and collagen II (Figure 8) expressions due to increases in the concentration of lead compound showed that the lead compound is a potential candidate for DMOAD development. 


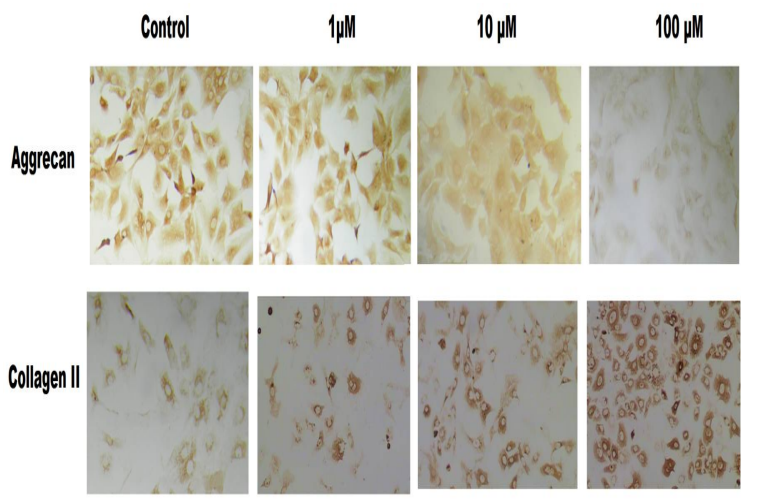

Figure 8: Effect of different concentrations of lead compound on the expression of aggrecan and collagen II in chondrocytes

\section{DISCUSSION}

OA has unknown pathophysiology affecting joints and leading to physical disability. In-silico techniques have been used to look for drugs for auto immune disorders like rheumatoid arthritis and OA $(21,22)$. Natural compounds have been treated as a goldmine to look for drugs for arthritis \{23]. OA currently lack treatment options [6] and looking for new strategies and compounds is the need of the hour. Xu et al showed compounds in Curcuma longa, Sophora japonica and Camellia sinensis modulate proinflammatory cytokines TNF- $\alpha$ and IL-1. Khan et al 2017 showed that Wogonin, a compound of natural origin modulates inflammation and shows chondroprotective effects. Chemical database (Maybridge) of 59000 compounds have been used for pharmacophore-based virtual screening to identify novel and potent ADAMTS-4 inhibitors, this study with its limitation to only docking-based studies have reported five possible ADAMTS-4 inhibitors (24). The use of natural compounds with both in-silico and in-vitro validation gives this study an advantage for further biological activity test.

\section{CONCLUSION}

The shortlisted lead compound can synergistically inhibit the S1 domain of MMP-13 protein. This study has successfully identified a natural compound that may pave the way for a novel DMOAD of natural origin against osteoarthritis.

\section{DECLARATIONS}

\section{Conflict of Interest}

No conflict of interest associated with this work.

\section{Contribution of Authors}

We declare that this work was done by the authors named in this article and all liabilities pertaining to claims relating to the content of this article will be borne by the authors. This study was designed by Yanzi Cheng. All the experiments were performed byYang Xiang, Yuanhui Li, Zhirong Ling.

\section{REFERENCES}

1. Krasnokutsky S, Samuels J, Abramson SB. Osteoarthritis in 2007. BULLETIN-HOSPITAL FOR JOINT DISEASES NEW YORK 2007; 65(3): 222.

2. Van den Berg W. Osteoarthritis year 2010 in review: pathomechanisms. Osteoarthritis Cartilage 2011; 19(4): 338-341.

3. Woolley DE. 6 - Cellular Mechanisms of Cartilage Destruction A2 - Henderson, B. In: Edwards JCW, Pettipher ER, editors. Mechanisms and Models in Rheumatoid Arthritis. London: Academic Press, 1995; pp 115-132.

4. Hollander AP, Pidoux I, Reiner A, Rorabeck C, Bourne R, Poole AR. Damage to type II collagen in aging and osteoarthritis starts at the articular surface, originates around chondrocytes, and extends into the cartilage with progressive degeneration. J Clin Invest 1995; 96(6): 2859-2869.

5. Poole AR, Kobayashi M, Yasuda T, Laverty S, Mwale F, Kojima T,Sakai T, Wahl C, El-Maadawy S, Webb G, Tchetina E, Wu W.I. Type II collagen degradation and its regulation in articular cartilage in osteoarthritis. Ann Rheum Dis 2002; 61(suppl 2)ii: 78-81.

6. Pelletier J-P, Martel-Pelletier J. DMOAD Developments. Bull NYU Hosp Jt Dis 2007; 65(3): 242-248.

7. Fosang AJ, Little CB. Drug insight: aggrecanases as therapeutic targets for osteoarthritis. Nat ClinPract Rheumatol 2008; 4(8): 420-427.

8. Goldring $M B$, Otero $M$. Inflammation in osteoarthritis. Curr Opin Rheumatol 2011; 23(5): 471-478.

9. Goldring $M B$. The role of the chondrocyte in osteoarthritis. Arthritis Rheum 2000; 43(9): 1916-1926.

10. Mitchell PG, Magna HA, Reeves LM, Lopresti-Morrow LL, Yocum SA, Rosner PJ, Geoghegan KF, Hambor JE. Cloning, expression, and type II collagenolytic activity of matrix metalloproteinase-13 from human osteoarthritic cartilage. J Clin Invest 1996; 97(3): 761-768.

11. Tchetina EV, Kobayashi M, Yasuda T, Meijers T, Pidoux I, Poole AR. Chondrocyte hypertrophy can be induced by a cryptic sequence of type II collagen and is accompanied by the induction of MMP-13 and collagenase activity: implications for development and arthritis. Matrix Biol 2007; 26(4): 247-258.

12. Nara $H$, Kaieda $A$, Sato $K$, Naito $T$, Mototani $H$, Oki $H$, Yamamoto $Y$, Kuno $H$, Santou $T$, Kanzaki $N$. et al. Discovery of Novel, Highly Potent, and Selective Matrix Metalloproteinase (MMP)-13 Inhibitors with a 1, 2, 4Trop J Pharm Res, November 2018; 17(11): 2261 
Triazol-3-yl Moiety as a Zinc Binding Group Using a Structure-Based Design Approach. J Med Chem 2017; 60(2): 608-626.

13. Lipinski CA. Lead-and drug-like compounds: the rule-offive revolution. Drug Discovery Today: Technologies 2004; 1(4): 337-341.

14. Morris GM, Huey R, Lindstrom W, Sanner MF, Belew RK, Goodsell DS, Olson AJ. AutoDock4 and AutoDockTools4: Automated docking with selective receptor flexibility. J Comput Chem 2009; 30(16): 27852791.

15. Hess B, Bekker H, Berendsen HJ, Fraaije JG. LINCS: a linear constraint solver for molecular simulations. Journal of computational chemistry 1997; 18(12): 14631472.

16. Hess B, Kutzner C, Van Der Spoel D, Lindahl E. GROMACS 4: algorithms for highly efficient, loadbalanced, and scalable molecular simulation. Journal of chemical theory and computation 2008; 4(3): 435-447.

17. SchuËttelkopf AW, Van Aalten DM. PRODRG: a tool for high-throughput crystallography of protein-ligand complexes. Acta Crystallographica Section D: Biological Crystallography 2004; 60(8): 1355-1363.

18. Essmann U, Perera L, Berkowitz ML, Darden T, Lee H, Pedersen LG. A smooth particle mesh Ewald method. The Journal of chemical physics 1995; 103(19): 8577 8593.
19. Kumari R, Kumar R, Lynn A. g_mmpbsa A GROMACS Tool for High-Throughput MM-PBSA Calculations. Journal of chemical information and modeling 2014; 54(7): 1951-1962.

20. Johns D, Athanasiou K. Growth factor effects on costal chondrocytes for tissue engineering fibrocartilage. Cell Tissue Res 2008; 333(3): 439-447.

21. Khan NM, Haseeb A, Ansari MY, Devarapalli $P$, Haynie $S$, Haqqi TM. Wogonin, a plant derived small molecule, exerts potent anti-inflammatory and chondroprotective effects through the activation of ROS/ERK/Nrf2 signaling pathways in human Osteoarthritis chondrocytes. Free Radical Biology and Medicine 2017; 106: 288-301.

22. Xu S, Peng H, Wang N, Zhao M. Inhibition of TNF- $\alpha$ and IL-1 by compounds from selected plants for rheumatoid arthritis therapy: In vivo and in silico studies. Tropical Journal of Pharmaceutical Research 2018; 17(2): 27785.

23. Khanna D, Sethi G, Ahn KS, Pandey MK, Kunnumakkara $A B$, Sung $B$, Aggarwal A, Aggarwal BB. Natural products as a gold mine for arthritis treatment. Current opinion in pharmacology 2007 1; 7(3): 344-51.

24. Verma $P$, Dalal $K$, Chopra $M$. Pharmacophore development and screening for discovery of potential inhibitors of ADAMTS-4 for osteoarthritis therapy. Journal of molecular modeling 2016 1; 22(8): 178. 